\title{
Fully 3D Bayesian Image Reconstruction for the ECAT EXACT HR+ ${ }^{1}$
}

\author{
Jinyi $\mathrm{Qi}^{2}$, Student Member, IEEE, Richard M. Leahy ${ }^{2}$, Member, IEEE, Chinghan Hsu ${ }^{2}$, Student Member, \\ IEEE, Thomas H. Farquhar ${ }^{3}$, Student Member, IEEE, and Simon R. Cherry ${ }^{3}$, Senior Member, IEEE \\ ${ }^{2}$ Signal and Image Processing Institute, University of Southern California \\ ${ }^{3}$ Crump Institute for Biological Imaging, UCLA School of Medicine
}

\begin{abstract}
A fully 3D Bayesian method is described for high resolution reconstruction of images from the Siemens/CTI ECAT EXACT $\mathrm{HR}+$ whole body positron emission tomography (PET) scanner. To maximize resolution recovery from the system we model depth dependent geometric efficiency, intrinsic detector efficiency, photon pair non-colinearity, crystal penetration and inter-crystal scatter. We also explicitly model the effects of axial rebinning and angular mashing on the detection probability or system matrix. By fully exploiting sinogram symmetries and using a factored system matrix and automated indexing schemes, we are able to achieve substantial savings in both the storage size and time required to compute forward and backward projections. Reconstruction times are further reduced using multi-threaded programming on a four processor Unix server. Bayesian reconstructions are computed using a Huber prior and a shifted-Poisson likelihood model that accounts for the effects of randoms subtraction and scatter. Reconstructions of phantom data show that the 3D Bayesian method can achieve improved FWHM resolution and contrast recovery ratios at matched background noise levels compared to both the 3D reprojection method and an OSEM method based on the shifted-Poisson model.
\end{abstract}

\section{INTRODUCTION}

Image reconstruction in fully $3 \mathrm{D}$ PET is routinely performed using either the 3D reprojection method (3DRP) [1] or a rebinning procedure in combination with 2D filtered backprojection (FBP) [2]. While these algorithms can be realized with relatively low computational cost, particularly so for the rebinning methods, the accuracy of the reconstructed images is limited by the approximations implicit in the line integral model on which the reconstruction formulae are based. In contrast, statistical methods can adopt arbitrarily accurate models for the mapping between the source volume and the sinograms. Furthermore, these methods can optimize performance in low count situations through explicit modeling of the statistical variability inherent in photon limited coincidence detection.

The ability of statistical approaches to more accurately model coincidence detection makes them an attractive alternative to the analytic approaches. However, iterative 3D reconstruction represents a daunting computational

\footnotetext{
${ }^{1}$ This work was supported by the National Cancer Institute under Grant No. R01 CA579794 and Grant No. R01 CA56655.
}

challenge due to the large number of lines of response (LORs) collected for each data set. Two important factors that limit the practicality of iterative $3 \mathrm{D}$ reconstruction algorithms are (i) the computer memory required to represent the detection probability or system matrix that contains the probabilities of detecting photons from each voxel at each detector pair; and (ii) the per-iteration cost associated with forward and backward projection. One approach to reducing both of these factors is to use a combination of Fourier rebinning and a 2D iterative reconstruction method [3].. The major limitation of this approach is that the rebinning step assumes that the data are true line integrals so that the potential for resolution recovery is reduced. Another way to avoid large memory requirements is to use on-the-fly computation in the forward and backward projection step, as is done routinely in filtered backprojection. This approach has limited appeal since it restricts our ability to accurately model the system. In order to allow maximum possible resolution recovery, we have concentrated on developing a 3D method based on directly processing the raw data from the scanner using a pre-computed system matrix.

The full size of the system matrix for a clinical whole body scanner is huge (see Table 2) but it can be efficiently stored by exploiting its sparseness and the high degree of symmetry $[4,5]$. Further reductions in size can be realized by using automated indexing schemes and a factored system model so that a spatially variant detector response can be included at little additional cost compared to a simple strip-integral model $[6,7]$. Here we present the results of applying a Bayesian method to the reconstruction of data from the Siemens/CTI ECAT EXACT HR+ whole body scanner [8]. This method is a modification of that described in [7] and uses the same factored system matrix structure. Using this structure we are able to reduce the stored size of the matrix to approximately 50MB. The method differs from that in [7] in two ways: first, we modify the system matrix to explicitly model the axial rebinning and angular mashing procedures that are applied in the ECAT HR+ to reduce the data size. Second, we use a modified statistical model for the data, the shifted-Poisson model in [9], that accounts for the presence of scatter in the data and for the increase in variance resulting from randoms subtraction. Using this same shifted-Poisson model we also develop a modified OSEM method [10] which can compensate for scatter and again models the effect of randoms subtraction on variance. To achieve further reductions in computation time, we have implemented the method using multi-threading techniques on a four processor server. 
In Section II we briefly review the factored system model that was introduced in [7] for 3D PET. We also describe how axial rebinning and angular mashing are included in this model. We then describe the statistical model for the data and image and present details of the reconstruction method in Section III. Results are presented in Section IV for line source measurements in a uniform background and also for various sized lesions in a thorax phantom. Finally we show a whole body example for a human volunteer and present our conclusions.

\section{SYSTEM MODEL}

Table 1

ECAT EXACT HR+ scanner parameters. The numbers in (.) are for the standard 3D mashing mode.

\begin{tabular}{|l|r|}
\hline Ring diameter, $\mathrm{mm}$ & 413.45 \\
\hline Detectors per ring & 576 \\
\hline Number of rings & 32 \\
\hline Angles per sinogram & $288(144)$ \\
\hline LORs per angle & 288 \\
\hline Max ring difference & 22 \\
\hline LORs per sinogram & $82,944(41,472)$ \\
\hline Number of sinograms & $934(239)$ \\
\hline Total LORs & $77 \times 10^{6}\left(9.9 \times 10^{6}\right)$ \\
\hline
\end{tabular}

Table 2

Stored size of rebinned system matrices for ECAT EXACT HR+ (excluding attenuation correction and detector normalization files).

\begin{tabular}{|l|r|r|}
\hline Object & Body object & Brain object \\
\hline FOV, $\mathrm{mm}^{3}$ & $577 \times 577 \times 155$ & $288 \times 288 \times 155$ \\
\hline Image size & $128 \times 128 \times 32$ & $128 \times 128 \times 63$ \\
\hline Voxel size, $\mathrm{mm}^{3}$ & $4.51 \times 4.51 \times 4.85$ & $2.25 \times 2.25 \times 2.43$ \\
\hline Full $\boldsymbol{P}$ & $5 \times 10^{12}$ & $10 \times 10^{12}$ \\
\hline $\boldsymbol{P}_{\text {Geom }}$ & 42 Mbytes & 56 Mbytes \\
\hline $\boldsymbol{P}_{\text {Blur }}$ & \multicolumn{2}{|c|}{$0.5 \mathrm{Mbytes}$} \\
\hline Total & 43 Mbytes & 57 Mbytes \\
\hline
\end{tabular}

Listed in Table 1 are the characteristics of the ECAT HR+ scanner [11]. In Table 2 we have listed the corresponding object and system matrix sizes. Clearly it is necessary to store the matrix in a sparse form and to exploit symmetries to reduce storage and computation costs. In [7] we described a factored matrix approach to efficiently storing the system matrix in 3D PET. We summarize its main properties here.

\section{A. Factorization of the Projection Matrix}

The elements, $p(i, j)$, of the detection probability matrix $\boldsymbol{P} \in \mathbb{R}^{M \times N}$ denote the probability of detecting an emission from pixel site $j, j=1 \ldots N$ at detector pair $i, i=1, \ldots M$. In order to reduce the storage size of the $\boldsymbol{P}$ matrix, we factor it as follows:

$$
\boldsymbol{P}=\boldsymbol{P}_{\text {det.sens }} \boldsymbol{P}_{\text {det.blur }} \boldsymbol{P}_{\text {attn }} \boldsymbol{P}_{\text {geom }}
$$

$\boldsymbol{P}_{\text {geom }}$ is the geometric projection matrix with each element $(i, j)$ equal to the probability that a photon pair produced in voxel $j$ reaches the front faces of the detector pair $i$ in the absence of attenuation and assuming perfect photon-pair colinearity. Our model incorporates a depth dependent geometric sensitivity that is calculated using the solid angle spanned by the voxel $j$ at the faces of the detector pair $i$ [7]. Although the full size of $\boldsymbol{P}_{\text {geom }}$ is extremely large, $\boldsymbol{P}_{\text {geom }}$ is very sparse and has redundancies of which we can take advantage to reduce the storage size. By choosing the voxel size in the $z$ direction to be an integer fraction of the ring distance, we have in-plane rotation symmetries, axial reflection symmetry, and parallel symmetry in $\boldsymbol{P}_{\text {geom }}$ as described in $[4,5,7]$. The non-zero elements of $\boldsymbol{P}_{\text {geom }}$ are stored using automated voxel indexing in a ray-driven projector format. In this way, we were able to reduce the storage requirement of $\boldsymbol{P}$ to the sizes shown in Table 2.

$\boldsymbol{P}_{\text {det.blur }}$ is the sinogram blurring matrix used to model photon pair non-colinearity, inter-crystal scatter and penetration [6]. In principle, a 3D sinogram blurring model should be used to model radial, angular and inter-sinogram blurring. In our current implementation, since the axial acceptance angle of the scanner is small $\left(\leq 10.3^{\circ}\right.$ when the maximum ring difference is set to 22 ), we have assumed that these blurring effects can be confined to a single sinogram and use a 2D blurring model. Furthermore, by ignoring effects associated with the location of each detector within the $8 \times 8$ blocks [6] and assuming that the blurring kernels are identical for sinograms for all ring differences, we then only need to compute and store the blurring kernels for the projection rays of a single projection angle. While this model assumes separability of depth dependent and sinogram blurring factors, our studies of point source sinogram data using 2D ECAT HR+ [6] and 3D microPET data [7] indicate that this approximation provides an accurate model of the photon detection process. $\boldsymbol{P}_{\text {det.blur }}$ is generated by Monte Carlo simulation with statistical modeling of the detector properties [6]. By factoring sinogram blurring out of the geometric projection matrix, we achieve a factor of 3 saving in the matrix size and comparable savings in reconstruction time.

$\boldsymbol{P}_{\text {attn }}$ is a diagonal matrix containing the attenuation coefficients. The blank and transmission scans are acquired in $2 \mathrm{D}$ rather than $3 \mathrm{D}$ mode due to dead time considerations. The $3 \mathrm{D}$ attenuation coefficients are then computed from the forward projection of a reconstructed $2 \mathrm{D}$ attenuation image. We use FBP reconstructions in phantom studies where the transmission scans are high count. However, for lower count situations, such as those encountered in whole body PET, a MAP method is used to reconstruction the transmission image [12] which can lead to substantial improvements in the accuracy of the attenuation factors as shown in [13].

$\boldsymbol{P}_{\text {det.sens }}$ is again a diagonal matrix which contains the detector efficiencies. Let detector pair $i$ consist of the $k$ th and $l$ th detector, then the efficiency of detector pair $i$ can be expressed as [14]

$$
\boldsymbol{P}_{\text {det.sens }}(i, i)=f_{k, l} * g_{k, l} * \epsilon_{k, l} * d_{k, l}
$$


where $f_{k, l}$ represents the radially varying geometric efficiencies due to the ring structure of the detectors; $g_{k, l}$ represents detector-pair geometric efficiencies due to non-equal detector surface areas within the block and other block effects; $\epsilon_{k, l}=\epsilon_{k} * \epsilon_{l}$ is the product of the intrinsic detector efficiencies which vary due to crystal imperfections, light guide variations, differences in photomultiplier (PMT) gains and variations in the electronics used to detect PMT signals; and finally, $d_{k, l}$ represents the non-uniform loss of counts due to dead-time, which are computed based on the singles rates of the detector buckets [14].

\section{B. Axial Rebinning and Angular Mashing}

The ECAT HR+ scanner used in our study acquires 3D data using axial rebinning and angular mashing. These procedures are used to reduce the data to a more manageable size and are justified on the grounds that the raw data set is oversampled. The rebinning procedure used in the scanner is very similar to that described in [15] and can best be understood with reference to their Fig. 1.

The rebinning is defined in terms of the number of detector rings $N_{r}$, the maximum ring difference $d_{\text {max }}$, and a span factor $S$. Let $\{Y\}_{m, n}$ denote the oblique sinogram collected between detector rings $m$ and $n, m, n=0,1, \ldots N_{r}-1$. The rebinned sinograms $\{Y\}_{p, q}^{R}$ are indexed by an oblique plane number $q=0,1,2, \ldots, 2 N_{r}-1$ and a segment number $p=0, \pm 1, \pm 2, \ldots,|p| \leq \frac{d_{\max }}{S}$. The rebinning procedure is then defined by the sum:

$$
\{Y\}_{p, q}^{R}=\sum_{(m, n) \in A_{p, q}}\{Y\}_{m, n}
$$

where

$$
A_{p, q}=\{(m, n): m+n=q,|m-n-p S| \leq(S-1) / 2\}
$$

Note that for some $(p, q)$, the set $A_{p, q}$ is empty and the corresponding $\{Y\}_{p, q}^{R}$ is not used in the reconstruction. In our studies, the maximum ring difference $d_{\text {max }}$ is set to 22 and the span $S$ to 9 . As a result, all the sinograms acquired were grouped into 5 segments for a total of 239 non-empty sinograms [11].

Angular mashing is used to further reduce the number of LORs by adding adjacent views, thus halving the number of samples in the azimuthal direction [11]. Let $k$ denote the angle index and $l$ the ray index, then angular mashing can be written as:

$$
\{Y\}_{p, q}^{R M}(k, l)=\{Y\}_{p, q}^{R}(2 k, l)+\{Y\}_{p, q}^{R}(2 k+1, l)
$$

In contrast to FBP methods based on the line integral model, the statistically based approach allows us to explicitly model these data compression procedures in our geometric projection matrix, $\boldsymbol{P}_{\text {geom }}$. To do this, we first compute $p_{\text {geom }}(i, j)$ for all ring differences, $d=0,1,2, \ldots, d_{\text {max }}=22$. We then add the $p_{\text {geom }}(i, j)$ 's to match the rebinning procedure in (3). Angular mashing is performed directly in each forward and backward projection, which enables us to utilize the same
$\boldsymbol{P}_{\text {geom }}$ matrix for different mashing factors, but results in some loss in computing efficiency. Studies performed using phantom data showed that accurately modeling the effects of rebinning and mashing in the system matrix, rather than simply pruning the matrix to delete the rows which are rebinned into others, produces improvements in image contrast recovery.

\section{IMAGE RECONSTRUCTION}

\section{A. The Statistical Model}

To model the effect of randoms subtraction and the presence of scatter in the data, we use a shifted-Poisson likelihood model [9]. The statistical model that we use for the image is a $3 \mathrm{D}$ Gibbs prior with a Huber potential function. The image is reconstructed by maximizing the log posterior density function:

$$
\begin{aligned}
\hat{\boldsymbol{x}}(Y)= & \arg \max _{\boldsymbol{x} \geq 0} L(Y \mid \boldsymbol{x})-\beta \phi(\boldsymbol{x}) \\
L(Y \mid \boldsymbol{x})= & \sum_{i}\left[-\left(\hat{Y}_{i}+2 r_{i}+s_{i}\right)\right. \\
& \left.+\left(Y_{i}+2 r_{i}\right) \log \left(\hat{Y}_{i}+2 r_{i}+s_{i}\right)\right] \\
\phi(\boldsymbol{x})= & \sum_{j} \sum_{\substack{k \in \mathcal{N}_{j} \\
k>j}} \kappa_{j k} V\left(x_{j}-x_{k}\right)
\end{aligned}
$$

where $Y$ are the projection data, $\beta$ is the hyperparameter of the Gibbs prior, $\hat{Y}$ is the projection associated with image $\boldsymbol{x}$, i.e. $\hat{Y}=\boldsymbol{P} \boldsymbol{x}$, and $r_{i}$ and $s_{i}$ are the estimated means of the randoms and scattered events, respectively.

The shifted Poisson model accounts for the increase in variance in the data due to randoms subtraction and the off-set in the mean due to the presence of scatter. This model requires estimates of the mean of the random and scattered events. Since the delayed counts that are used for randoms correction are not routinely acquired separately in the ECAT HR+ scanner, we estimate the randoms mean from the total number of counts in the delayed-coincidence window. If we assume that the singles flux is uniform at all detectors, then variations in randoms are due solely to differences in the intrinsic detector efficiencies and dead-time. Using this assumption we compute $r_{i}$ as

$$
r_{i}=N_{d} \frac{n_{i}}{\sum_{j} n_{i}}
$$

where

$$
n_{i}=\epsilon_{k, l} * d_{k, l}
$$

$N_{d}$ is the total count of events in the delayed window and $k, l$ are the detector indices that corresponded to the detector pair $i$. Note that we do not include geometric efficiencies and block effects here since these effects apply only to true coincidence pairs.

The scatter component, $s_{i}$, is estimated using the single-scatter simulation technique described in $[16,17]$. We use the implementation of this scatter computation method supplied by CTI.

The Huber potential function $V\left(x_{j}-x_{k}\right)$ is defined as

$$
V\left(x_{j}-x_{k}\right)=\left\{\begin{array}{cl}
\frac{1}{2 \delta_{h}}\left(x_{j}-x_{k}\right)^{2}, & \text { if }\left|x_{j}-x_{k}\right| \leq \delta_{h} \\
\left|x_{j}-x_{k}\right|-\frac{\delta_{h}}{2}, & \text { otherwise }
\end{array}\right.
$$


where $\delta_{h}$ is a small constant (we used $1 \%$ of the estimated maximum image value). The neighborhood $\mathcal{N}_{j}$ we used here is the third order (26-neighbors) neighborhood with $\kappa_{j k}$ equal to the reciprocal of the distance between the two voxels.

\section{B. Computing the MAP Estimate}

Using the factored system matrix approach and exploiting the symmetries in the geometric projection matrix, we gain substantial savings in both storage and computational requirements. We note however that to fully realize this saving we must consider all data and all voxels at each iteration. Therefore we adopt a preconditioned conjugate gradient (PCG) approach:

$$
\begin{aligned}
\boldsymbol{x}^{(n+1)} & =\boldsymbol{x}^{(n)}+\alpha^{(n)} \boldsymbol{a}^{(n)} \\
\boldsymbol{a}^{(n)} & =\boldsymbol{d}^{(n)}+\beta^{(n-1)} \boldsymbol{a}^{(n-1)} \\
\boldsymbol{d}^{(n)} & =\boldsymbol{C}^{(n)} \boldsymbol{g}^{(n)} \\
\beta^{(n-1)} & =\frac{\left(\boldsymbol{g}^{(n)}-\boldsymbol{g}^{(n-1)}\right)^{\prime} \boldsymbol{d}^{(n)}}{\boldsymbol{g}^{(n-1)^{\prime}} \boldsymbol{d}^{(n-1)}}
\end{aligned}
$$

where $g^{(n)}$ is the gradient vector of the log posterior density function at $\boldsymbol{x}=\boldsymbol{x}^{(n)}$ and $\boldsymbol{C}^{(n)}$ is the preconditioner:

$$
C^{(n)}=\operatorname{diag}\left\{\frac{x_{j}^{(n)}+\delta}{\sum_{i} p(i, j)}\right\}
$$

The step size $\alpha^{(n)}$ is found using a Newton-Raphson line search. The $\delta$ is a small positive number to ensure that $C^{(n)}$ is positive definite. Here we set $\delta$ equal to $0.01 \max _{j}\left\{x_{j}^{(n)}\right\}$.

The algorithm is initialized with a constant image which when forward projected matches the total number of counts in the data after subtracting the scatter sinogram. The search vector is initialized by setting $\boldsymbol{a}^{(0)}=\boldsymbol{d}^{(0)}$. At each iteration we test whether the search vector is an ascent direction, i.e.

$$
\boldsymbol{g}^{(n)^{\prime}} \boldsymbol{a}^{(n)}>0
$$

If not, then we reinitialize the PCG algorithm with $\boldsymbol{a}^{(n)}=\boldsymbol{d}^{(n)}$.

The PCG algorithm is modified in the following way in order to impose a non-negativity constraint on the image. The line search in (10) is performed without directly constraining the image, but with the weaker constraint that the forward projection of the image be non-negative. After the line search is completed the updated image in (10) is truncated by setting any negative values to zero, i.e. we form the intermediate image vector $\hat{\boldsymbol{x}}^{(n+1)}=T\left\{\boldsymbol{x}^{(n)}+\alpha^{(n)} \boldsymbol{a}^{(n)}\right\}$ where $T\{$.$\} is$ the truncation operator. Finally, to ensure an increase in the posterior density, we perform a second line search along the direction $\left(\hat{\boldsymbol{x}}^{(n+1)}-\boldsymbol{x}^{n}\right)$ to form the updated image vector:

$$
\boldsymbol{x}^{(n+1)}=\boldsymbol{x}^{(n)}+\gamma^{(n)}\left(\hat{\boldsymbol{x}}^{(n+1)}-\boldsymbol{x}^{(n)}\right), 0<\gamma^{(n)} \leq 1
$$

where $\gamma^{(n)}$ is chosen to maximize the posterior density. Because the line search is less time consuming than forward and backward projection, this extra line search has little effect on total computational cost.

\section{Modified Ordered subsets EM Reconstruction}

For comparative purposes, we also derived an OSEM algorithm for the shifted Poisson model with scatter. The log likelihood function is given in (6). It can be shown that

$$
\frac{\partial L(\boldsymbol{x})}{\partial x_{j}}=-\sum_{i} p(i, j)+\sum_{i} \frac{\left(Y_{i}+2 r_{i}\right) p(i, j)}{\sum_{l} p(i, l) x_{l}+2 r_{i}+s_{i}}
$$

With analogy to the relationship between the standard Poisson model and the EM and OSEM algorithms, we propose the following modified OSEM update equation:

$$
x_{j}^{k+1}=\frac{x_{j}^{k}}{\sum_{i \in S_{k}} p(i, j)} \sum_{i \in S_{k}} \frac{\left(Y_{i}+2 r_{i}\right) p(i, j)}{\sum_{l} p(i, l) x_{l}^{k}+2 r_{i}+s_{i}}
$$

where $S_{k}$ is the $k$ th subset of the projection data. Eq. (15) is repeated until all the subsets have been exhausted. In the following, one iteration refers to one cycle through all of the data subsets. Note that (15) becomes the standard OSEM method in [10] when $r_{i}$ and $s_{i}$ are zero. We found that including $r_{i}$ in (15) provides some improvement in contrast recovery in the thorax phantom study when compared to the standard OSEM method.

Only a subset of the data are used in each update step (15). Consequently, the 2D sinogram blurring matrix can not be used efficiently here. By assuming that the projection data vary slowly from one view to the next, we approximate the detector response using a $1 \mathrm{D}$ radial-only blurring kernel. The $1 \mathrm{D}$ radial blurring factors were obtained by summing the $2 \mathrm{D}$ blurring matrix over all the angles of view. In the studies presented below, the projection data were grouped into 9 subsets. Each subset consists of projection views separated by $11.25^{\circ}$ degrees.

\section{Computation Time}

A multi-threaded version of the MAP reconstruction algorithm has been implemented on a Sun Ultra Enterprise 4000 server with four $168 \mathrm{MHz}$ processors. One fully 3D iteration using all four processors for a body sized object takes about 7.5 mins compared to 25 mins for a single processor on the same system. Benchmark comparisons with the 300 $\mathrm{MHz}$ Pentium II processor indicate that reconstruction times of 60 mins are achievable for 20 iterations of the MAP method using a relatively low cost four processor Pentium-II system. It is difficult to partition the data for the OSEM algorithm to fully exploit symmetries in the geometric matrix so that one complete cycle through the data using OSEM takes approximately 1.5 times that for MAP. However, since the number of iterations required is usually lower, we expect the total reconstruction time for OSEM to be on the order of $50 \%$ or less of that for the MAP algorithm.

\section{RESULTS}

Phantom studies were performed to compare performance of the 3D MAP algorithm with the 3D OSEM and 3DRP methods. All the data were acquired with the ECAT HR+ high resolution tomograph in standard 3D mode: maximum 
ring difference of 22, a span of 9, and a angular mashing factor of 2. The 3DRP reconstruction code we used was that supplied by CTI. Scatter correction in 3DRP was performed by subtracting the scatter sinogram from the projection data before reconstruction.

Each of the three methods tested uses one variable, collectively referred to below as the smoothing parameter, to effect a trade-off between resolution and background noise variance. In the MAP reconstructions, the hyperparameter $\beta$ in (6) was varied to achieve different resolutions. In the OSEM method, smoothness was controlled using the number of iterations. For the 3DRP method, a Hann windowing filter with different cutoff frequencies was used in both axial and transaxial directions. In addition, we used a ramp filter with a cutoff equal to the Nyquist frequency to achieve the highest possible resolution.

\section{A. Cylinder and Line Source Study}

A $20 \mathrm{~cm}$ diameter uniform cylinder and three axiallyoriented line sources were activated with FDG solution in the ratio of 1:500 per unit volume. The diameter of the line sources was approximately $0.5 \mathrm{~mm}$. The emission data were collected for 5 minutes with a total of 6.4 million counts. The transmission data were collected 14 hours after the emission scan for a total of 60 minutes. The long duration transmission scan was performed to minimize artifacts and noise due to errors in the attenuation correction factors. All reconstruction methods used the same 3D attenuation coefficients computed from the 2D transmission and blank scans using the standard protocol with CTI software.

The emission image was reconstructed using the MAP, OSEM and 3DRP methods. Fig. 1 shows the reconstructed images and profiles of a transaxial slice at $4 \mathrm{~cm}$ off center in the axial direction. Fig. 2(a) shows the contrast between the reconstructed line source and background vs. the standard deviation of the background; Fig. 2(b) shows the FWHM of the line source vs. the standard deviation of the background. The MAP and OSEM reconstructions were computed using a voxel size of $2.3 \times 2.3 \times 4.85 \mathrm{~mm}^{3}$. The 3 DRP images were first reconstructed on a voxel size of $2.3 \times 2.3 \times 2.425 \mathrm{~mm}^{3}$ and then the adjacent image planes were added together to match the MAP and OSEM images. Since the voxel size is far larger than the line source diameter, partial volume effects should be considered when interpreting these plots. The transaxial FWHMs were calculated as the diameter of a circle with area equal to the image area for which the transaxial point spread function exceeds half of its maximum value. The background standard deviations were estimated using the same transaxial slice as the spatial standard deviation of the reconstructions in a $15 \mathrm{~cm}$ diameter circular region that excluded the line sources; these values were then normalized by the mean of the same background.

The results in Figs. 1 and 2 show that the statistical methods can achieve higher contrast recovery and smaller FWHM than 3DRP method at matched noise levels. Although the FWHMs of MAP and OSEM are quite similar, the MAP method consistently produces better contrast which may translate to improved lesion
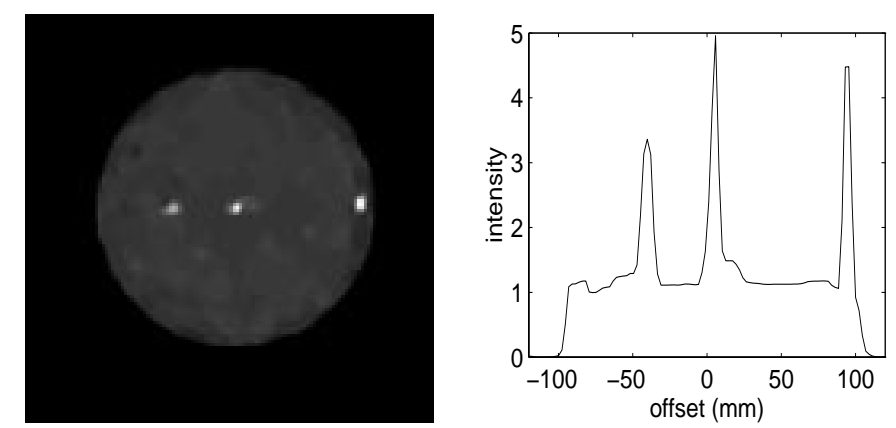

(a)
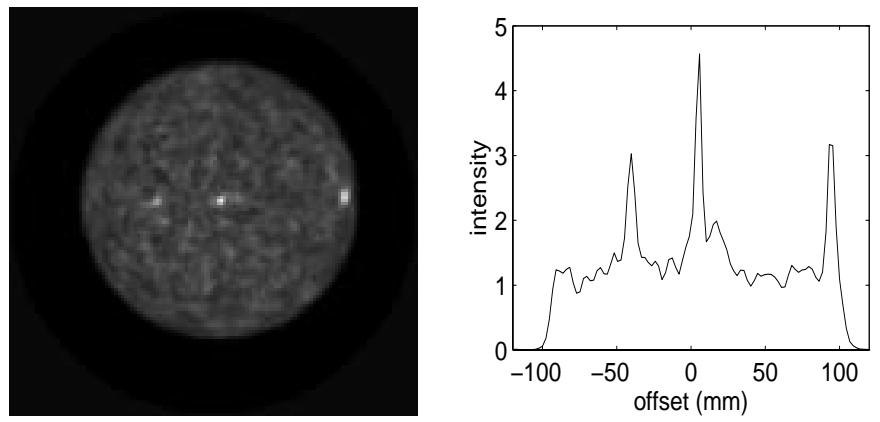

(b)
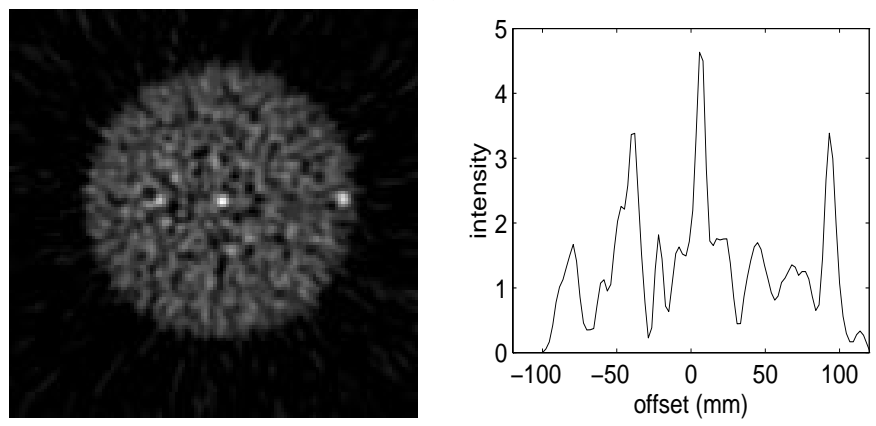

(c)

Fig. 1 Reconstructed images (left column) and profiles (right column) of the line sources in a uniform cylindrical background: (a) MAP, (b) OSEM, and (c) 3DRP. The smoothing parameters for the three methods were chosen to achieve approximately matched contrast $(\approx 3)$ for the line source at the center of the field of view using the curves in Fig. 2.

detectability.

\section{B. Thorax Phantom Study}

A thorax phantom was used to study lesion detectability through computation of contrast recovery coefficients for different sized "lesions." The phantom contains thorax, lung and heart compartments. The heart has three chambers: an inner cylinder, surrounded by concentric middle and outer chambers. The inner heart chamber was filled with water. The lungs were filled with a lung-equivalent foam. The thorax wall and the middle and outer heart chambers were activated with ${ }^{18} \mathrm{~F}$ in the ratio of approximately $1: 3$ per unit volume. Four different size lesions $(0.2 \mathrm{ml}, 0.45 \mathrm{ml}, 1.0 \mathrm{ml}$, and $1.9 \mathrm{ml})$ were activated with ${ }^{13} \mathrm{NH}_{3}$ and put in the thorax chamber at different axial locations. The ratio between the intensity of the lesions and the thorax wall was 12:1 at the time of scanning.

The total counts in the emission scan was 14 million which 

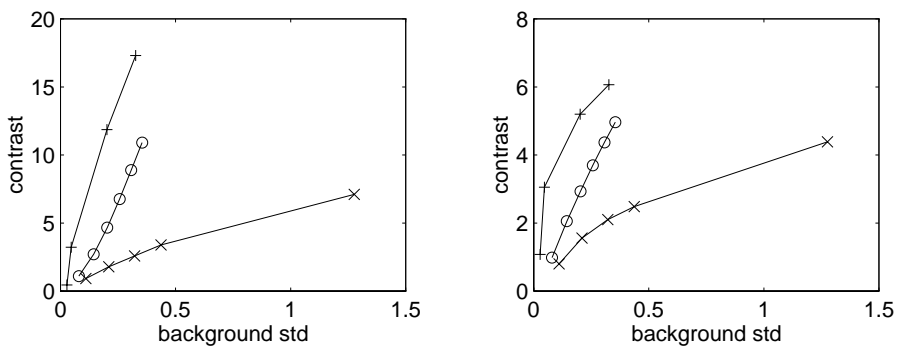

(a)
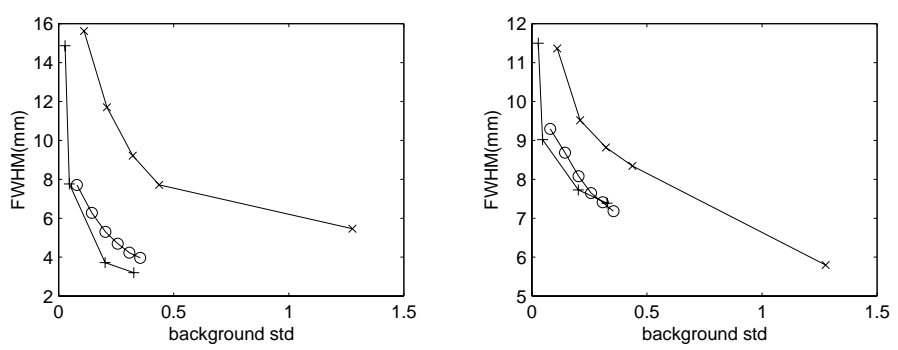

(b)

Fig. 2 Comparison of contrast recovery and transaxial FWHM for the line sources reconstructed using MAP ('+'), OSEM ('o'), and 3DRP (' $X$ '). Left column: centered line source; right column: $95 \mathrm{~mm}$ off-center line source.

is similar to that for a clinical whole body study of the thorax. A 2D transmission scan was collected for 120 minutes. The 3D attenuation factors were computed using CTI software. The emission image was reconstructed using MAP and OSEM with voxel sizes of $4.51 \times 4.51 \times 4.85 \mathrm{~mm}^{3}$ and 3DRP with a voxel of size $4.51 \times 4.51 \times 2.425 \mathrm{~mm}^{3}$. Again adjacent image planes in the 3DRP reconstructions were added together to match the MAP and OSEM voxel sizes.

Fig. 3 shows transaxial slices through the volume at the levels of the $0.2 \mathrm{ml}$ and $1.0 \mathrm{ml}$ lesions. The corresponding profiles are shown in Fig. 4. In Fig. 5 we plot the contrast recovery coefficients (CRC) of the lesions versus the spatial variance of the reconstructed thorax wall. The CRC is defined as:

$$
C R C=\frac{\max _{\text {lesion }} / \text { mean }_{\mathrm{bg}}-1}{\text { true }_{\text {lesion }} / \text { true }_{\mathrm{bg}}-1}
$$

The mean and variance of the background were calculated from 5 selected circular regions on the thorax wall. The average was taken over the center slice that contains the maximum value of the lesion and two adjacent slices for a total of 570 voxels.

The results obtained from the thorax phantom study again show the power of the statistical methods in achieving higher contrast recovery at matched noise levels compared to the 3DRP method. The MAP method also outperforms OSEM in contrast recovery. Note that the CRCs of the 3DRP reconstructions are not affected by the sum of adjacent planes except for the $0.45 \mathrm{ml}$ lesion which appears to be located at the edge of two adjacent planes. The small improvements in contrast recovery for this case when using the smaller voxels is offset by an increase in background variance which is not reflected in Fig. 5.

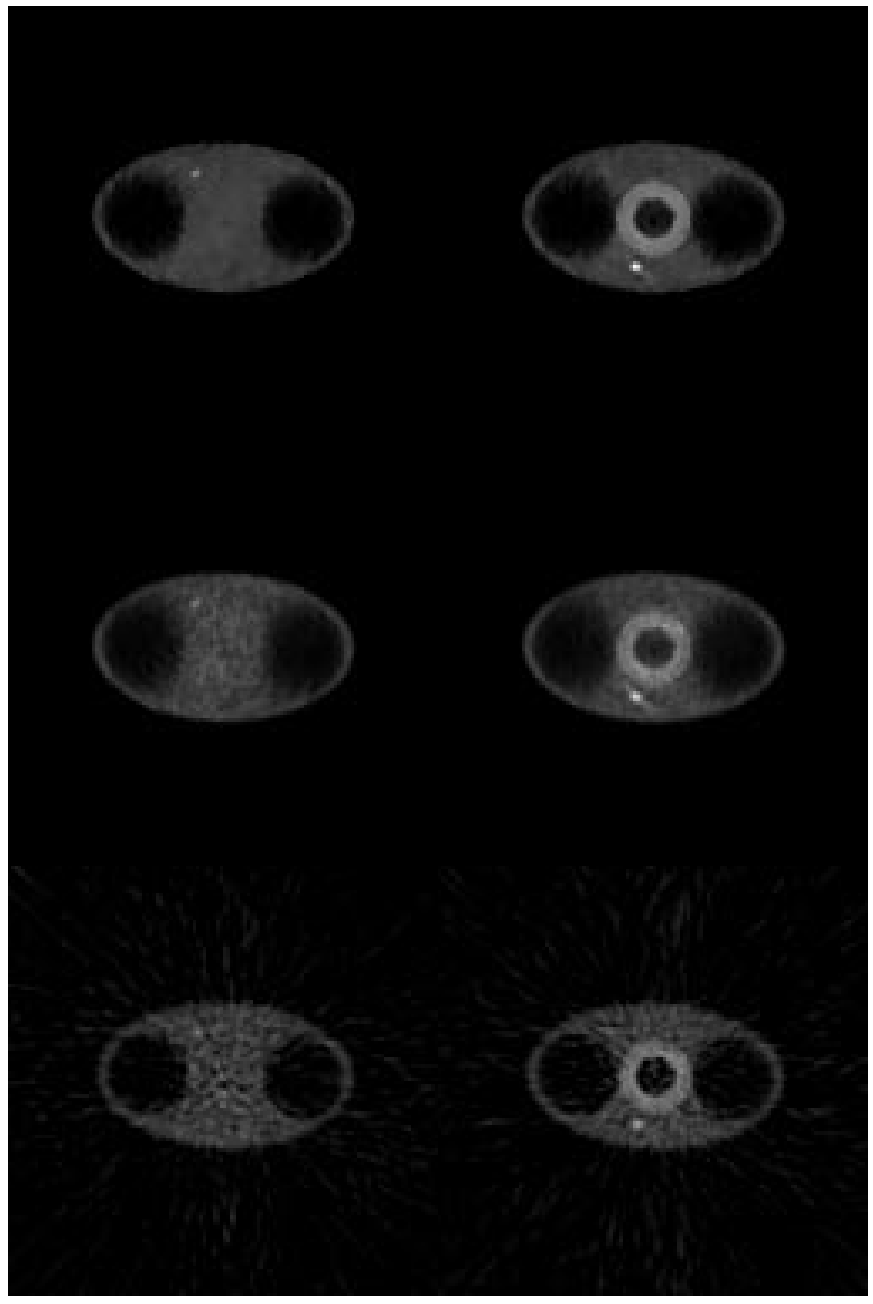

Fig. 3 Transaxial planes through the reconstructed thorax phantom showing the $0.2 \mathrm{ml}$ lesion (left column) and $1.0 \mathrm{ml}$ lesion (right column): top row: MAP; middle row: OSEM; and bottom row: 3DRP. The smoothing parameters were selected to have matched CRC $(\approx 0.15)$ for the $0.2 \mathrm{ml}$ lesion using the curves in Fig. 5.

\section{Normal Volunteer Study}

We conclude with an example of an FDG whole body scan of a normal volunteer. The emission scan duration was 1.5 minutes per bed position with a total of 5 bed positions. The total counts were about 11.4 million per bed position. Transmission scans were collected for 3 minutes per bed position before injection. Fig. 6 shows coronal slices through the reconstructed transmission and emission images for the different reconstruction algorithms. The attenuation correction factors used in the 3DRP images were computed using CTI software which is based on FBP reconstructions of the attenuation images. MAP and OSEM images used attenuation factors based on a reprojection of a Bayesian reconstruction of the attenuation image. The OSEM and MAP images show reduced artifacts and improved contrast recovery in the heart when compared to all of the 3DRP images. The MAP image shows smoother background than that in the OSEM image but it is difficult to draw strong conclusions from this single example, particularly since no pathology is present. 


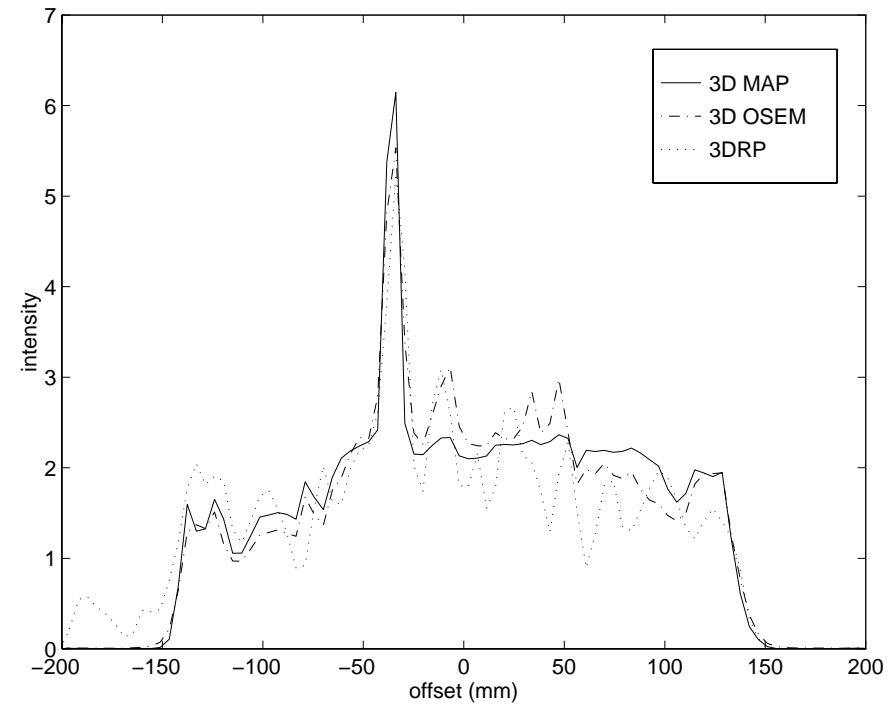

(a)

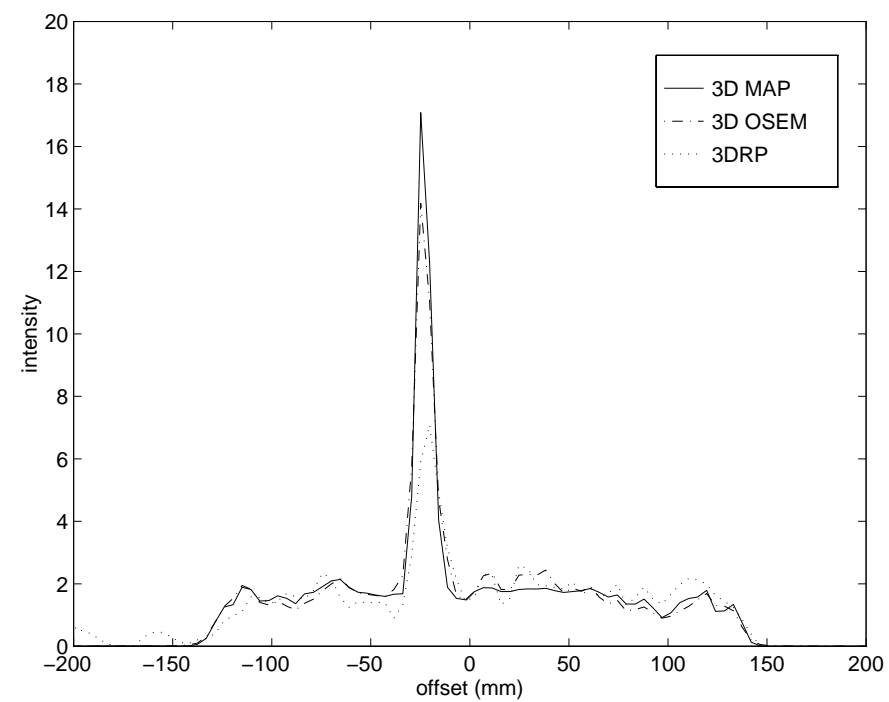

(b)

Fig. 4 Profiles of the reconstructed thorax phantom for (a) $0.2 \mathrm{ml}$ lesion and (b) $1.0 \mathrm{ml}$ lesion. Profiles are taken along the horizontal line passing through the maximum of each lesion.

\section{CONCLUSION}

We have described a fully 3D MAP reconstruction method for the ECAT HR+ scanner that can be easily modified for other clinical multi-ring PET scanners. We have shown that by using the factored system matrix approach and exploiting symmetries we gain substantial savings in both storage and computation requirements. Using a multi-threaded implementation of this method, a $128 \times 128 \times 32$ body sized image can be reconstructed in about 1 hour using a $4-\mathrm{CPU} 300 \mathrm{MHz}$ Pentium-II server. Line source and thorax phantom studies show that this MAP method can achieve higher resolution and contrast recovery than OSEM and 3DRP methods at matched background noise levels.

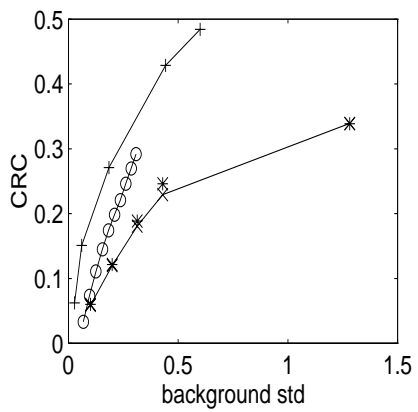

(a)

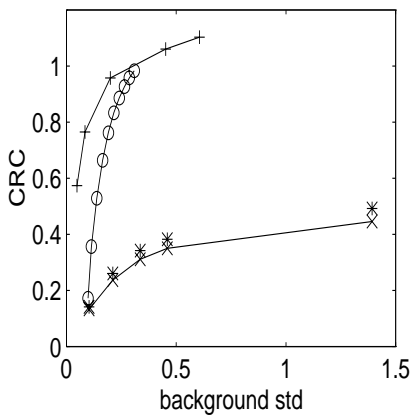

(c)

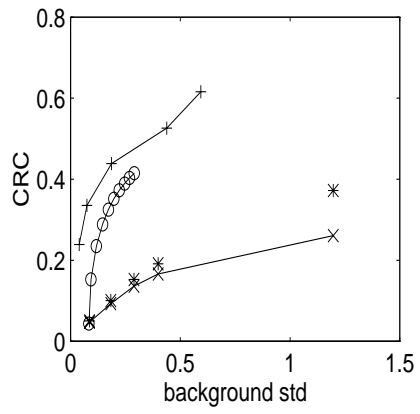

(b)

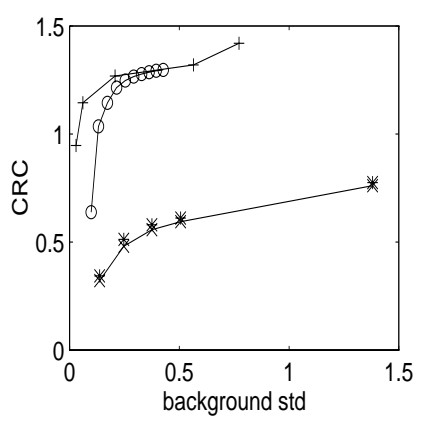

(d)
Fig. 5 Comparison of CRCs of the lesions reconstructed using MAP ('+'), OSEM ('o'), and 3DRP (' $\times$ '). The '*'s indicate the CRCs achieved using 3DRP before adjacent image planes are summed together. (a) $0.2 \mathrm{ml}$ lesion, (b) $0.45 \mathrm{ml}$ lesion, (c) $1.0 \mathrm{ml}$ lesion, and (d) $1.9 \mathrm{ml}$ lesion.

\section{ACKNOWLEDGMENTS}

The authors would like to thank E. U. Mumcuoglu and N. Doshi for help with the Siemens/CTI ECAT EXACT HR+ file formats and associated software.

\section{REFERENCES}

[1] P. Kinahan and J. Rogers, "Analytic 3D image reconstruction using all dectected events," IEEE Transactions on Nuclear Science, vol. 36, pp. 964-968, 1989.

[2] M. Defrise, P. E. Kinahan, D. W. Townsend, C. Michel, M. Sibomana, and D. F. Newport, "Exact and approximate rebinning algorithms for 3-D PET data," IEEE Transactions on Medical Imaging, vol. 16, no. 2, pp. 145-158, April 1997.

[3] P. Kinahan, C. Michel, and M. Defrise, "Fast iterative image reconstruction of 3D PET data," in Proc. IEEE Nuclear Science Symposium and Medical Imaging Conference, Anaheim, CA, 1996, pp. 1918-1922.

[4] C. Johnson, Y. Yan, R. Carson, R. Martino, and M. DaubeWitherspoon, "A system for the 3D reconstruction of retractedsepta PET data using the EM algorithm," IEEE Transactions on Nuclear Science, vol. 42, no. 4, pp. 1223-1227, August 1995.

[5] C. Chen, S. Lee, and Z. Cho, "Parallelization of the EM algorithm for 3D PET image reconstruction," IEEE Transactions on Medical Imaging, vol. 110, pp. 513-522, 1991.

[6] E. Mumcuoglu, R. Leahy, S. Cherry, and E. Hoffman, “Accurate geometric and physical response modeling for statistical image reconstruction in high resolution PET," in Proc. IEEE Nuclear Science Symposium and Medical Imaging Conference, 1996, pp. 1569-1574. 


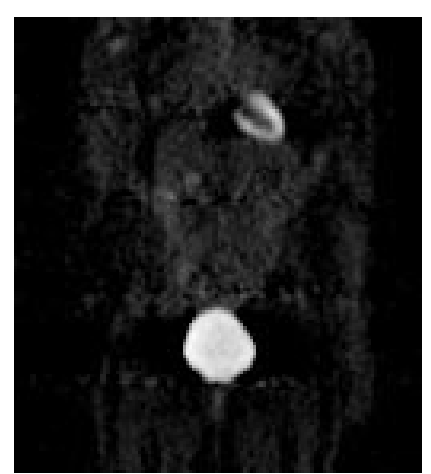

(a)

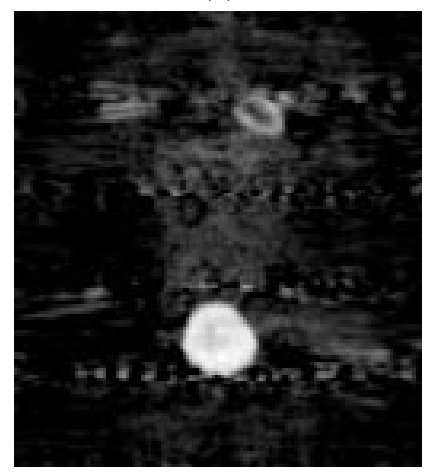

(c)

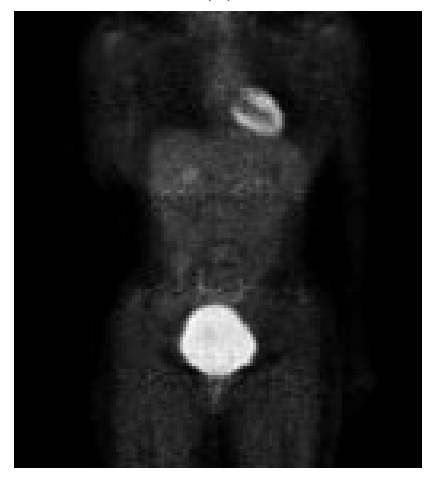

(e)

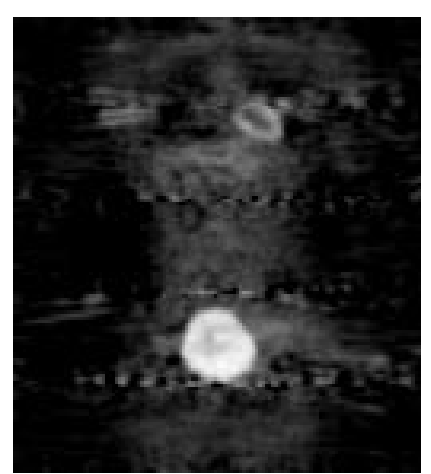

(b)

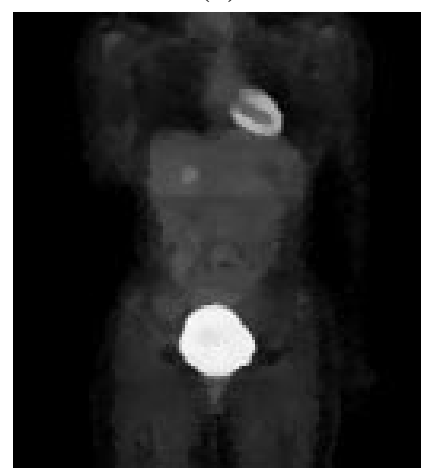

(d)

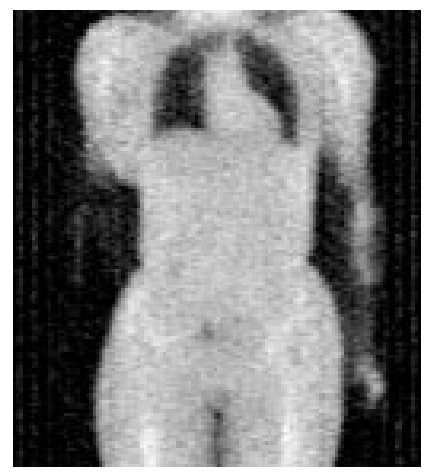

(f)
Fig. 6 The reconstructed whole body image: (a) 3DRP, without attenuation and scatter correction; (b) 3DRP, with attenuation correction but without scatter correction; (c) 3DRP, with attenuation and scatter correction; (d) MAP; (e) OSEM; (f) 2D MAP reconstruction of transmission image.

[7] J. Qi, R. M. Leahy, S. R. Cherry, A. Chatziioannou, and T. H. Farquhar, "High resolution 3D bayesian image reconstruction using the microPET small animal scanner," to appear in Physics in Medicine and Biology, March 1998.

[8] Manufactured by CTI PET System, Inc., Knoxville, TN 37932, USA.

[9] M. Yavuz and J. A. Fessler, "Objective functions for tomographic reconstruction from randoms-precorrected PET scans," in Proc. IEEE Nuclear Science and Medical Imaging Conference, 1996, vol. 2, pp. 1067-1071.

[10] H. M. Hudson and R. S. Larkin, "Accelerated image reconstruction using ordered subsets of projection data," IEEE Transactions on Medical Imaging, vol. 13, no. 4, pp. 601-609, 1994.

[11] L.-E. Adam, J. Zaers, H. Ostertag, H. Trojan, M. E. Bellemann, and G. Brix, "Performance evaluation of the whole-body PET scanner ECAT EXACT HR+ following the IEC standard," IEEE Trans. Nucl. Sci., vol. 44, no. 3, pp. 1172-1179, June 1997.

[12] E. Mumcuoglu, R. Leahy, S. Cherry, and Z. Zhou, "Fast gradientbased methods for Bayesian reconstruction of transmission and emission PET images," IEEE Transactions on Medical Imaging, vol. 13, no. 4, pp. 687-701, December 1994.

[13] E. Mumcuoglu, R. Leahy, and S. Cherry, "Bayesian reconstruction of PET images: Methodology and performance analysis," Physics in Medicine and Biology, vol. 41, pp. 1777-1807, 1996.

[14] M. E. Casey, H. Gadagkar, and D. Newport, "A component based method for normalization in volume PET," in 3rd International Meeting on Fully 3D Reconstruction in Nuclear Medicine and Radiology, Aix-les-Bains, 1995, pp. 67-71.

[15] T. Bruckbauer, K. Wienhard, S. B. Hansen, L. Eriksson, and G. Blomquist, "Evaluation of the ECAT EXACT HR with ACSII for clinical routine 3D measurements," in Proc. IEEE Nuclear Science Symposium and Medical Imaging Conference, San Francisco, CA, October 1995, vol. 3, pp. 1378-1382.

[16] C. C. Watson, D. Newport, and M. E. Casey, "A single scatter simulation technique for scatter correction in 3D PET," in $3 \mathrm{rd}$ International Meeting on Fully 3D Reconstruction in Nuclear Medicine and Radiology, Aix-les-Bain, France, 1995.

[17] C. C. Watson, D. Newport, M. E. Casey, R. A. deKemp, R. S. Beanlands, and M. Schmand, "Evaluation and simulation based scatter correction for 3D PET cardiac imaging," IEEE Transactions on Nuclear Science, vol. 44, pp. 90-97, 1997. 